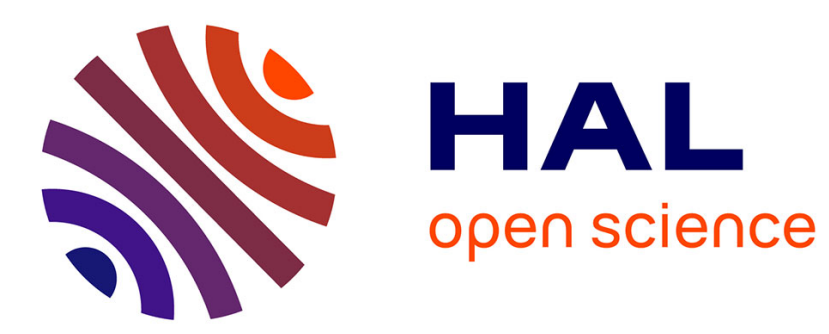

\title{
Hydrodynamic Coefficients and Wave Loads for a WEC Device in Heaving Mode
}

Sébastien Olaya, Jean-Matthieu Bourgeot, Mohamed Benbouzid

\section{To cite this version:}

Sébastien Olaya, Jean-Matthieu Bourgeot, Mohamed Benbouzid. Hydrodynamic Coefficients and Wave Loads for a WEC Device in Heaving Mode. 2013 MTS/IEEE OCEANS, Jun 2013, Bergen, Norway. pp.1-8. hal-00874394

\section{HAL Id: hal-00874394 https://hal.science/hal-00874394}

Submitted on 17 Oct 2013

HAL is a multi-disciplinary open access archive for the deposit and dissemination of scientific research documents, whether they are published or not. The documents may come from teaching and research institutions in France or abroad, or from public or private research centers.
L'archive ouverte pluridisciplinaire HAL, est destinée au dépôt et à la diffusion de documents scientifiques de niveau recherche, publiés ou non, émanant des établissements d'enseignement et de recherche français ou étrangers, des laboratoires publics ou privés. 


\section{Hydrodynamic Coefficients and Wave Loads for a WEC Device in Heaving Mode}

\author{
Sébastien Olaya \\ École Nationale d'Ingénieurs de Brest École Nationale d'Ingénieurs de Brest \\ EA 4325 LBMS \\ Email : olaya@enib.fr \\ Jean-Matthieu Bourgeot \\ EA 4325 LBMS \\ Email : bourgeot@enib.fr
}

\author{
Mohamed Benbouzid \\ University of Brest \\ EA 4325 LBMS
}

Email : Mohamed.Benbouzid@univ-brest.fr

\begin{abstract}
This paper deals with the hydrodynamic parameter computation of a Wave Energy Converter (WEC) that consists of a cylindrical buoy sliding along a partially submerged platform made up of a plate and a column. The computed parameters are particularly needed for the development of a simple hydrodynamic time-dependant model based on the Cummins formulation. This model is intended to be used for WEC control purposes. A semi-analytical approach is therefore proposed for the computation of the hydrodynamic coefficients and the excitation forces. The boundary value problem is solved using variable separation and matched eigenfunction expansion methods. Analytical expressions for the velocity potential are then obtained for each subdomain. Using afterwards these expressions enables the hydrodynamic coefficients and the excitation force to be computed. Numerical results are given for different radiuses of the buoy, column and plate and are compared with previously published models.
\end{abstract}

Index Terms-Wave Energy Converter, potential theory, eigenfunction expansion, wave-loads, heaving mode, scattering and radiation problem.

\section{INTRODUCTION}

This work was motivated from the need to provide a set of hydrodynamic parameters for the development of a simple hydrodynamic time-dependant model based on the Cummins formulation for a Wave Energy Converter (WEC) [1]. This time domain model is needed to analyse the WEC behavior in irregular waves. The WEC device schematically depicted on Fig. 1 consists of a cylindrical buoy riding in waves and sliding along a partially submerged platform made up of a plate and a column. Energy resulting from the relative motion between the two concentric bodies is extracted from the incoming wave by a gearbox and an electric generator. The present paper focuses on the determination of the excitation forces and hydrodynamic coefficients (i.e. added mass and radiation damping) for heaving mode in water of finite depth. Due to the simplicity of the geometry and in view of the model purpose (i.e. optimisation and control) these parameters are evaluated using a semi-analytical approach which is propably the fastest and most reliable way to get them. From the literature review, it has been noticed that in [2], Berggren and Johansson (1992) have treated the radiation problem of a wave energy device consisting of a buoy connected to a submerged plate by an elastometric hose. The buoy and plate have the same radius. They presented results for added mass and potential

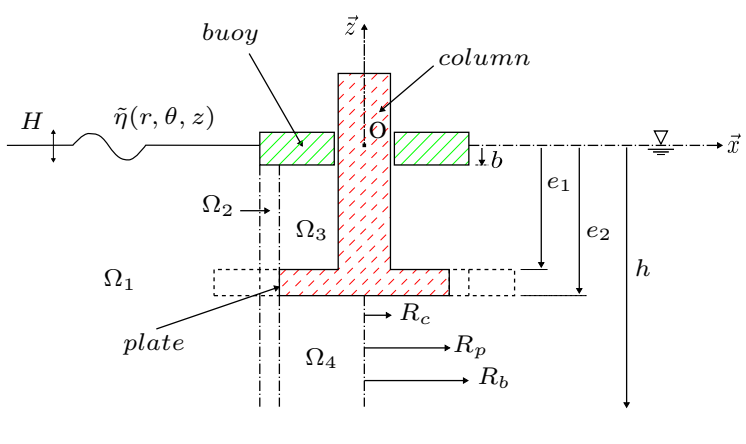

Fig. 1. Definition sketch of the Wave Energy Converter.

damping for the two bodies and included cross terms only for the heaving mode. Under the assumption of linear wave theory, the radiation problem is solved by using the method of eigenfunction expansions. A comparison is made between their solution and a solution proposed by Yeung R.W. (1981) [3] who studied the whole radiation problem of a floating vertical cylinder. A similar study was performed in [4] where the buoy is rigidly connected to a submerged vertical tube in which a plate acts as a piston. In addition to the radiation problem, the author has presented results for the excitation forces obtained through the Haskind's theorem. Wu et al. investigated in [5] hydrodynamic properties of a similar WEC where the buoy riding in waves is directly connected to a caisson fixed on the seabed by a rigid rope. Wave energy is harnessed by a liquid pump in the caisson through the rope. In this paper the radius of the caisson is greater than or equal to that of the buoy. In [6], the same authors extended the solution to the case where the caisson radius is smaller than that of the buoy. They presented analytical results for the response amplitudes and hydrodynamic forces for different radius sizes of the submerged structure and the gap between them. It is shown that the buoy hydrodynamic behaviour becomes more complex when the caisson radius is large and only at relatively low frequency. Also it seems that the resonance period does not vary regardless of the increase or decrease of the radius ratio and/or the gap. In [7] a similar study was performed with a two-body moving structure but their analysis where restricted to the case where the radius of the submerged cylinder is larger than or equal to that of the floating one. 
A set of theoretical hydrodynamic parameters is presented and a similar behavior was found as the previous study for the buoy over the caisson. More recently some hydrodynamic results have been reported in [8] and [9] for the UC Berkeley wave energy device. The main idea is to extract energy due to the relative heaving motion between two concentric surfacepiercing truncated circular cylinders. A similar study was first performed by Mavrakos (2004) in [10]. Throughout the literature review, it is worth noting that there are no analytical results for the particular WEC configuration given in Fig. 1.

\section{Mathematical Formulation of the Problem}

Following the well-known procedure for that kind of resolution, we divided the whole fluid domain into three or four subdomains depending on the plate radius size as indicated in Fig. 1. Considering a cylindrical coordinate system $(r, \theta, z)$ with its origin $O$ located at the intersection of the undisturbed free surface level with cylinders axes and the $z$-axis is positive upward it follows

- region $\Omega_{1}: r \geq R_{x} ;-h \leq z \leq 0$

where $R_{x}=\max \left(R_{b}, R_{p}\right)$

- region $\Omega_{2}$

- For $R_{p}>R_{b}: R_{b} \leq r \leq R_{p} ;-e_{1} \leq z \leq 0$

- For $R_{p}=R_{b}:$ does not exist

- For $R_{p}<R_{b}: R_{p} \leq r \leq R_{b} ;-h \leq z \leq-b$

- region $\Omega_{3}: R_{c} \leq r \leq R_{x} ;-e_{1} \leq z \leq-b$

where $R_{x}=\min \left(R_{b}, R_{p}\right)$

- region $\Omega_{4}: r \leq R_{p} ;-h \leq z \leq-e_{2}$

$R_{b}$ is the radius of the buoy, $R_{p}$ is the radius of the plate, $R_{c}$ is the radius of the column and $h$ is the water depth not necessarily infinite. Others geometrical parameters are given in Fig. 1.

Assuming linear wave theory and supposing that the fluid is inviscid, incompressible, and its motion is irrotational, fluid flow can be described, using a complex representation, by the velocity potential

$$
\Phi(r, \theta, z, t)=\operatorname{Re}\left\{\phi(r, \theta, z) e^{-\mathrm{i} \omega t}\right\}
$$

where $\operatorname{Re}\{\}$ denotes the real part of the complex expression, $\omega$ is the angular frequency, $t$ is the time dependency. Also lets defined the free surface elevation as

$$
\eta(r, \theta, z, t)=\operatorname{Re}\left\{\tilde{\eta}(r, \theta, z) e^{-\mathrm{i} \omega t}\right\}
$$

which is related to the velocity potential by the linearised Free Surface Kinematic Boundary Condition (FSKBC) (3)

$$
\frac{\partial \phi}{\partial z}=\frac{\partial \eta}{\partial t}, \quad \text { on } z=0
$$

and the linearised Free Surface Dynamic Boundary Condition (FSDBC) (4)

$$
\frac{\partial \phi}{\partial t}+g \eta=0, \quad \text { on } z=0
$$

The spatial part of the velocity potential $\phi(r, \theta, z)$ has to satisfy the following boundary value problem
- Governing equation

$$
\Delta \phi=0, \quad \text { in the whole fluid domain }
$$

- Free surface boundary condition from (3) and (4)

$$
\omega^{2} \phi-\left.g \frac{\partial \phi}{\partial z}\right|_{z=0}=0
$$

- Seabed boundary condition

$$
\left.\frac{\partial \phi}{\partial z}\right|_{z=-h}=0
$$

- Body surface boundary condition

$$
\nabla \phi \cdot \vec{n}=\vec{U}_{k} \cdot \vec{n}, \quad \text { on } S_{k}, k=1,2
$$

where $g$ is the acceleration of gravity, $\vec{n}$ the unit normal vector on wet body surfaces $S_{k}$ directed into the fluid domain, and $\vec{U}_{k}$ is the body velocity. $k=\{1,2\}$ stands respectively for the buoy and for the plate. Finally the velocity potentials $\phi$ must satisfy conditions specifying that the wave propagates away from the structure. This is given by the so-called Sommerfeld radiation condition expressed in $\Omega_{1}$ as

$$
\lim _{r \rightarrow+\infty} \sqrt{r}\left(\frac{\partial \phi}{\partial r}-i k_{e} \phi\right)=0
$$

where $k_{e}$ is the wave number.

From the linear water wave theory and according to [1], the velocity potential can be decomposed as

$$
\phi(r, \theta, z)=\phi_{0}(r, \theta, z)+\phi_{7}(r, \theta, z)+\sum_{k=1}^{2} \sum_{q=1}^{6} \phi_{q}^{k}(r, \theta, z)
$$

where $\phi_{0}$ is the incident waves potential, $\phi_{7}$ is the diffracted potentiel, and $\phi_{q}^{k}$ is the radiated potential due to the motion of the body $k$ in the direction $q$, with $q=\{1,3,5\}$ standing respectively for the surge, heave, and pitch mode of motion. In the solution procedure, analytical expressions for velocity potentials are obtained using the variables separation method Assuming angular independency due to the axysymmetric configuration, velocity potentials are expressed as infinite series of orthogonal functions into each subdomain fulfilling all boundary conditions. More details can be found on mathematical formulations in [1], [11], [12]. In the following, a solution to the boundary value problem will be presented, but for the sake of simplicity only for the case where the plate and buoy have the same radius.

\section{A. Radiated Potential for Each Subdomain in Heaving Mode}

The radiation problem corresponds to the case where the structure is forced to oscillate in the absence of incident waves. Following a procedure similar to the one presented in [10], we can decompose the velocity potential in each subdomain, $\phi_{q}^{l, k}(r, \theta, z)$ as follows

$$
\phi_{q}^{l, k}(r, \theta, z)=\sum_{m=0}^{\infty}-\mathrm{i} \omega \zeta_{q} \varphi_{q, m}^{l, k}(r, z) \cos (m \theta)
$$

where $l$ denotes the fluid domain and $\zeta_{q}$ is the complex amplitude corresponding to the mode of motion. For heaving mode 
( $q=3$ ) only expressions for $m=0$ gave a contribution [13] and then we are looking for solutions that can be expressed as

$$
\varphi_{3,0}^{l, k}(r, z)=\varphi_{3, h}^{l, k}(r, z)+\varphi_{3, p}^{l, k}(r, z)
$$

where $\varphi_{3, p}^{l, k}(r, z)$ represents a particular solution of the velocity potential in heaving mode that fulfills the inhomogeneous boundary conditions and $\varphi_{3, h}^{l, k}(r, z)$ is the homogeneous part of the solution to the boundary value problem.

In region $\Omega_{1}$, the velocity potential can be expressed as

$\varphi_{3, h}^{1, k}(r, z)=A_{0}^{k} \frac{H_{0}^{(1)}\left(k_{e} r\right)}{H_{0}^{(1)}\left(k_{e} R_{x}\right)} Z_{0}^{1}(z)+\sum_{i=1}^{\infty} A_{i}^{k} \frac{K_{0}\left(\lambda_{i} r\right)}{K_{0}\left(\lambda_{i} R_{x}\right)} Z_{i}^{1}(z)$

where $R_{x}=\max \left(R_{b}, R_{p}\right) . A_{i}^{k}$ are the unknown Fourier coefficients, $H_{0}^{(1)}()$ are Hankel functions and $K_{0}^{(1)}()$ are modified Bessel functions of the second kind.

For convenience we define $\lambda_{0}=-\mathrm{i} k_{e}$ then the dispersion relation is given in terms of the eigenvalues $\lambda_{i}$ by

$$
\omega^{2}=k_{e} g \tanh \left(k_{e} h\right)=-\lambda_{i} g \tan \left(\lambda_{i} h\right)
$$

and the depth dependency function $Z_{i}^{1}(z)$ is

$$
Z_{i}^{1}(z)=N_{\lambda_{i}}^{-1 / 2} \cos \left(\lambda_{i}(z+h)\right)
$$

where

$$
N_{\lambda_{i}}=\frac{1}{2}\left[1+\frac{\sin \left(2 \lambda_{i} h\right)}{2 \lambda_{i} h}\right]
$$

for $i=0,1, \cdots, \infty$

In region $\Omega_{3}$, the velocity potential can be expressed as

$$
\varphi_{3, h}^{3, k}(r, z)=\sum_{n=0}^{\infty}\left[D_{n}^{k} T_{n}(r)+E_{n}^{k} \tilde{T}_{n}(r)\right] Z_{n}^{3}(z)
$$

Expressions for the functions $T_{n}(r), \tilde{T}_{n}(r)$ are defined in Appendix $\mathrm{A}$ in terms of modified Bessel functions of the first and second kind, and $D_{n}^{k}, E_{n}^{k}$ are the unknown Fourier coefficients. The depth dependency function $Z_{n}^{3}(z)$ is defined as

$$
Z_{n}^{3}(z)= \begin{cases}1, & \text { for } n=0 \\ \sqrt{2} \cos \left(\alpha_{n}\left(z+e_{1}\right)\right), & \text { for } n \geq 1\end{cases}
$$

with

$$
\alpha_{n}=\frac{n \pi}{e_{1}-b_{1}}
$$

for $n=1,2, \cdots, \infty$.

From the body surface condition (8) we have

$$
\begin{aligned}
& \left.\frac{\partial \varphi_{3}^{3, k}}{\partial z}\right|_{z=-b}=\delta_{1 k}, \text { for } R_{c} \leq r \leq R_{x} \\
& \left.\frac{\partial \varphi_{3}^{3, k}}{\partial z}\right|_{z=-e_{1}}=\delta_{2 k}, \text { for } R_{c} \leq r \leq R_{x}
\end{aligned}
$$

where $R_{x}=\min \left(R_{b}, R_{p}\right), \delta_{i j}$ is the Kronecker's delta defined as $\delta_{i j}=1$ if $i=j$, and $\delta_{i j}=0$ otherwise.

Then the particular solution is given by

$$
\begin{aligned}
\varphi_{3, p}^{3, k}(r, z)= & \frac{1}{2\left(e_{1}-b\right)}\left[\left(z+e_{1}\right)^{2}-\frac{r^{2}}{2}\right] \delta_{1 k} \\
& -\frac{1}{2\left(e_{1}-b\right)}\left[(z+b)^{2}-\frac{r^{2}}{2}\right] \delta_{2 k}
\end{aligned}
$$

Finally for region $\Omega_{4}$ we have

$$
\varphi_{3, h}^{4, k}(r, z)=F_{0}^{k}+\sum_{j=1}^{\infty} F_{j}^{k} \frac{I_{0}\left(\beta_{j} r\right)}{I_{0}\left(\beta_{j} R_{p}\right)} Z_{j}^{4}(z)
$$

where $I_{0}^{(1)}()$ are modified Bessel functions of the first kind and $F_{j}^{k}$ are the unknown Fourier coefficients. The depth dependency function $Z_{j}^{4}(z)$ is defined as

$$
Z_{j}^{4}(z)= \begin{cases}1, & \text { for } j=0 \\ \sqrt{2} \cos \left(\beta_{j}\left(z+e_{2}\right)\right), & \text { for } j \geq 1\end{cases}
$$

with

$$
\beta_{j}=\frac{j \pi}{h-e_{2}}
$$

for $j=1,2, \cdots, \infty$.

From the body surface condition (8) we have

$$
\left.\frac{\partial \varphi_{3}^{4, k}}{\partial z}\right|_{z=-e_{2}}=\delta_{2 k}, \text { for } 0 \leq r \leq R_{p}
$$

and then the particular solution is expressed as

$$
\varphi_{3, p}^{4, k}(r, z)=\frac{1}{2(h-e 2)}\left[(z+h)^{2}-\frac{r^{2}}{2}\right] \delta_{2 k}
$$

\section{B. Expression of the Scattering Potential in Each Subdomain}

The scattering potential represented the solution for which the structure is considered fixed in presence of incident waves [11] and is defined as

$$
\phi_{s}(r, \theta, z)=\phi_{0}(r, \theta, z)+\phi_{7}(r, \theta, z)
$$

Considering a linear wave propagating in the positive direction $x$ in a water of constant depth $h$ and decribed by a small amplitude $A$ at frequency $\omega$, incident potential $\phi_{0}$ can be defined as

$$
\phi_{0}(r, \theta, z)=B \sum_{m=0}^{\infty} \epsilon^{m_{1}{ }^{m}} J_{m}\left(k_{e} r\right) Z_{0}^{1}(z) \cos (m \theta)
$$

where $\epsilon^{m}$ is the Neuman symbol defined as $\epsilon^{m}=1$ for $m=0$ and $\epsilon^{m}=2$ otherwise, $J_{m}()$ are Bessel functions of the first kind and $i$ is the complex number. The coefficient $B$ is defined by

$$
B=-\mathrm{i} A \frac{g}{\omega} \frac{1}{Z_{0}^{1}(0)}
$$

Following the same approach as the radiation problem, we are looking for unknown functions expressed as

$$
\phi_{s}^{l}(r, \theta, z)=\sum_{m=0}^{\infty} \varphi_{s, m}^{l}(r, z) \cos (m \theta)
$$


where only expressions for $m=0$ have a contribution in the vertical direction. From the gradient condition (8) we have at all body surfaces [1]

$$
\frac{\partial \varphi_{s}}{\partial n}=0
$$

Then it follows that the scattering velocity potential in region $\Omega_{1}$ can be expressed as

$$
\begin{aligned}
\varphi_{s, 0}^{1}(r, z)= & B J_{0}\left(k_{e} r\right) Z_{0}^{1}(z)+A_{0}^{0} \frac{H_{0}^{(1)}\left(k_{e} r\right)}{H_{0}^{(1)}\left(k_{e} R_{x}\right)} Z_{0}^{1}(z) \\
& +\sum_{i=1}^{\infty} A_{i}^{0} \frac{K_{0}\left(\lambda_{i} r\right)}{K_{0}\left(\lambda_{i} R_{x}\right)} Z_{i}^{1}(z)
\end{aligned}
$$

where $R_{x}=\max \left(R_{b}, R_{p}\right)$.

One can note that the expression given above has the same form as expression (12) obtained for the radiation problem. Then for the analytical resolution procedure purpose, let us introduce the notation $k=0$, which means none of the body is moving. Using notations given for radiation problem we can write

$$
\varphi_{s, 0}^{1}(r, z)=\varphi_{3, h}^{1,0}(r, z)+\varphi_{3, p}^{1,0}(r, z)
$$

where we define

$\varphi_{3, h}^{1,0}(r, z)=A_{0}^{0} \frac{H_{0}^{(1)}\left(k_{e} r\right)}{H_{0}^{(1)}\left(k_{e} R_{x}\right)} Z_{0}^{1}(z)+\sum_{i=1}^{\infty} A_{i}^{0} \frac{K_{0}\left(\lambda_{i} r\right)}{K_{0}\left(\lambda_{i} R_{x}\right)} Z_{i}^{1}(z)$

$\varphi_{3, p}^{1,0}(r, z)=B J_{0}\left(k_{e} r\right) Z_{0}^{1}(z)$

For others regions, unknown functions have the same forms as those developped for the homogeneous part of the radiation problem, then

$$
\varphi_{s, 0}^{l}(r, z)=\varphi_{3, h}^{l, 0}(r, z)
$$

for $l>1$.

It remains then to determine the unknown Fourier coefficients $A_{i}^{k}, D_{n}^{k}, E_{n}^{k}$, and $F_{j}^{k}$ in the infinite series of orthogonal functions using the matching eigenfunction expansion method. The coefficients can be determined by imposing conditions of pressure continuity and normal velocity at different imaginary interfaces (i.e. at $r=R_{b}$ and $r=R_{p}$ ) as well as the body surface boundary condition at the body vertical walls.

\section{Solution to the Boundary Value Problem}

In the case where the buoy radius and plate radius are equal, i.e. $R_{p}=R_{b}$, the requirement for pressure continuity and normal velocity has to be fulfilled at the vertical imaginary interface $r=R_{b}$, as well as the boundary conditions on the body vertical surfaces. For heaving mode the latter are expressed as

$$
\begin{aligned}
& \left.\frac{\partial \varphi_{3}^{1, k}}{\partial r}\right|_{r=R_{p}}=0, \text { for }-e_{2} \leq z \leq-e_{1} \\
& \left.\frac{\partial \varphi_{3}^{1, k}}{\partial r}\right|_{r=R_{b}}=0, \text { for }-b \leq z \leq 0 \\
& \left.\frac{\partial \varphi_{3}^{3, k}}{\partial r}\right|_{r=R_{c}}=0, \text { for }-e_{1} \leq z \leq-b
\end{aligned}
$$

for $k=0,1$ and 2 .

At the imaginary interface $r=R_{b}$, velocity potential continuity conditions are

- for $-e_{1} \leq z \leq-b$

$$
\varphi_{3}^{1, k}\left(R_{b}, z\right)=\varphi_{3}^{3, k}\left(R_{b}, z\right)
$$

$$
\left.\frac{\partial \varphi_{3}^{1, k}}{\partial r}\right|_{r=R_{b}}=\left.\frac{\partial \varphi_{3}^{3, k}}{\partial r}\right|_{r=R_{b}}
$$

- for $-h \leq z \leq-e_{2}$

$$
\varphi_{3}^{1, k}\left(R_{b}, z\right)=\varphi_{3}^{4, k}\left(R_{b}, z\right)
$$

$$
\left.\frac{\partial \varphi_{3}^{1, k}}{\partial r}\right|_{r=R_{b}}=\left.\frac{\partial \varphi_{3}^{4, k}}{\partial r}\right|_{r=R_{b}}
$$

Due to the orthogonal properties of the function $Z_{j}^{4}(z)$ valid for $-h \leq z \leq-e_{2}$, it follows that

$$
F_{j}^{k}=\frac{1}{h-e_{2}} \int_{-h}^{-e_{2}} \varphi_{3, h}^{4, k}\left(R_{b}, z\right) Z_{j}^{4}(z) \mathrm{d} z-Q_{j 3}^{k}+O_{j 3}^{k}
$$

where

$$
\begin{aligned}
& Q_{j 3}^{k}= \begin{cases}0, & \text { for } k=0 \\
\frac{1}{h-e_{2}} \int_{-h}^{-e_{2}} \varphi_{3, p}^{4, k}\left(R_{b}, z\right) Z_{j}^{4}(z) \mathrm{d} z, & \text { for } k=1,2\end{cases} \\
& O_{j 3}^{k}= \begin{cases}\frac{1}{h-e_{2}} \int_{-h}^{-e_{2}} \varphi_{3, p}^{1, k}\left(R_{b}, z\right) Z_{j}^{4}(z) \mathrm{d} z, & \text { for } k=0 \\
0, & \text { for } k=1,2\end{cases}
\end{aligned}
$$

Applying continuity condition (38), Fourier coefficients $F_{j}^{k}$ that describe velocity potential in $\Omega_{4}$ can be expressed in terms of coefficients $A_{i}^{k}$ as

$$
F_{j}^{k}=\sum_{i=0}^{\infty} A_{i}^{k} L_{j i}-\left(Q_{j 3}^{k}-O_{j 3}^{k}\right)
$$

with

$$
L_{j i}=\frac{1}{h-e_{2}} \int_{-h}^{-e_{2}} Z_{j}^{4}(z) Z_{i}^{1}(z) \mathrm{d} z
$$

Using the same procedure for interval $-e_{1} \leq z \leq-b$, orthogonal properties of the function $Z_{n}^{3}(z)$ lead to coefficients $D_{n}^{k}$

$$
D_{n}^{k}=\frac{1}{e_{1}-b} \int_{-e_{1}}^{-b} \varphi_{3, h}^{3, k}\left(R_{b}, z\right) Z_{n}^{3}(z) \mathrm{d} z-P_{n 3}^{k}+O_{n 3}^{k}
$$


where

$$
\begin{aligned}
& P_{n 3}^{k}= \begin{cases}0, & \text { for } k=0 \\
\frac{1}{e_{1}-b} \int_{-e_{1}}^{-b} \varphi_{3, p}^{3, k}\left(R_{b}, z\right) Z_{n}^{3}(z) \mathrm{d} z, & \text { for } k=1,2\end{cases} \\
& O_{n 3}^{k}= \begin{cases}\frac{1}{e_{1}-b} \int_{-e_{1}}^{-b} \varphi_{3, p}^{1, k}\left(R_{b}, z\right) Z_{n}^{3}(z) \mathrm{d} z, & \text { for } k=0 \\
0, & \text { for } k=1,2\end{cases}
\end{aligned}
$$

Applying continuity condition (36), Fourier coefficients $D_{n}^{k}$ that describe velocity potential in $\Omega_{3}$ can be expressed in terms of coefficients $A_{i}^{k}$ as

$$
D_{n}^{k}=\sum_{i=0}^{\infty} A_{i}^{k} M_{n i}-\left(P_{n 3}^{k}-O_{n 3}^{k}\right)
$$

with

$$
M_{n i}=\frac{1}{e_{1}-b} \int_{-e_{1}}^{-b} Z_{n}^{3}(z) Z_{i}^{1}(z) \mathrm{d} z
$$

Now by multiplying both sides of equations (33), (34), (37) and (39) by $Z_{\tau}^{1}(z) / h$ (for $\tau=0,1, \cdots, i, \cdots$ ), integrating over the corresponding interval of validity and adding resulting expressions, we get a complete set of equations. Replacing coefficients $F_{j}^{k}$ and $D_{n}^{k}$ by their respective definition (41) and (44) we obtain

$$
h_{\tau}^{k}=\sum_{i=0}^{\infty} d_{\tau i} A_{i}^{k}+\sum_{n=0}^{\infty} d_{\tau n} E_{n}^{k}
$$

with

$$
\begin{aligned}
h_{\tau}^{0}= & B\left(\Upsilon_{0} \delta_{0 \tau}-J_{0}\left(k_{e} R_{b}\right)\left[\frac{h-e_{2}}{h} \sum_{j=0}^{\infty} \Gamma_{j} L_{j 0} L_{j \tau}\right.\right. \\
& \left.\left.+\frac{e_{1}-b}{h} \sum_{n=0}^{\infty} T_{n}^{\prime}\left(R_{b}\right) M_{n 0} M_{n \tau}\right]\right) \\
h_{\tau}^{1}= & \frac{R_{b}}{2 h} M_{0 \tau}+\frac{e_{1}-b}{h} \sum_{n=0}^{\infty} T_{n}^{\prime}\left(R_{b}\right) P_{n 3}^{1} M_{n \tau} \\
h_{\tau}^{2}= & \frac{R_{b}}{2 h}\left(L_{0 \tau}-M_{0 \tau}\right)+\frac{h-e_{2}}{h} \sum_{j=0}^{\infty} \Gamma_{j} Q_{j 3}^{2} L_{j \tau} \\
& +\frac{e_{1}-b}{h} \sum_{n=0}^{\infty} T_{n}^{\prime}\left(R_{b}\right) P_{n 3}^{2} M_{n \tau} \\
d_{\tau i}= & \Delta_{i} \delta_{i \tau}+\frac{h-e_{2}}{h} \sum_{j=0}^{\infty} \Gamma_{j} L_{j i} L_{j \tau} \\
& +\frac{e_{1}-b}{h} \sum_{n=0}^{\infty} T_{n}^{\prime}\left(R_{b}\right) M_{n i} M_{n \tau} \\
d_{\tau n}= & \frac{e_{1}-b}{h} \sum_{n=0}^{\infty} \tilde{T}_{n}^{\prime}\left(R_{b}\right) M_{n \tau}
\end{aligned}
$$

where we defined

$$
\begin{aligned}
\Gamma_{0} & =0 & \Gamma_{j} & =\beta_{j} \frac{I_{1}\left(\beta_{j} R_{b}\right)}{I_{0}\left(\beta_{j} R_{b}\right)} \\
\Delta_{0} & =k_{e} \frac{H_{1}^{(1)}\left(k_{e} R_{b}\right)}{H_{0}^{(1)}\left(k_{e} R_{b}\right)} & \Delta_{i} & =\lambda_{i} \frac{K_{1}\left(\lambda_{i} R_{b}\right)}{K_{0}\left(\lambda_{i} R_{b}\right)}
\end{aligned}
$$

for $i, j \geq 1$ and

$$
\Upsilon_{0}=-k_{e} J_{1}\left(k_{e} R_{b}\right)
$$

Finally, multiplying the body surface condition (35), expressed on the column, by $Z_{\nu}^{3}(z) /\left(e_{1}-b\right)$ (for $\left.\nu=0,1, \cdots, n, \cdots\right)$, integrating with respect to $z$, i.e. $-e_{1} \leq z \leq-b$, and using the definition of $D_{n}^{k}$, we obtained the last set of equations at the vertical boundary $r=R_{c}$.

$$
h_{\nu}^{k}=\sum_{i=0}^{\infty} d_{\nu i} A_{i}^{k}+\sum_{n=0}^{\infty} d_{\nu n} E_{n}^{k}
$$

where

$$
\begin{aligned}
h_{\nu}^{0} & =-B J_{0}\left(k_{e} R_{c}\right) \sum_{n=0}^{\infty} T_{n}^{\prime}\left(R_{c}\right) M_{n 0} \delta_{n \nu} \\
h_{\nu}^{1} & =\sum_{n=0}^{\infty} T_{n}^{\prime}\left(R_{c}\right) P_{n 3}^{1} \delta_{n \nu}+\frac{R_{c}}{2\left(e_{1}-b\right)} \delta_{0 \nu} \\
h_{\nu}^{2} & =\sum_{n=0}^{\infty} T_{n}^{\prime}\left(R_{c}\right) P_{n 3}^{2} \delta_{n \nu}-\frac{R_{c}}{2\left(e_{1}-b\right)} \delta_{0 \nu} \\
d_{\nu i} & =\sum_{n=0}^{\infty} T_{n}^{\prime}\left(R_{c}\right) M_{n i} \delta_{n \nu} \\
d_{\nu n} & =\tilde{T}_{n}^{\prime}\left(R_{c}\right) \delta_{n \nu}
\end{aligned}
$$

In order to find a solution to Fourier coefficients we have to truncate the infinite series to the first $N$ terms. Thereby by introducing matrix notation, the unknown coefficients can be determined solving simultaneously the two sets of equations (46) and (55) that can be expressed by the following linear system.

$$
\left[\begin{array}{ll}
d_{\tau i} & d_{\tau n} \\
d_{\nu i} & d_{\nu n}
\end{array}\right]\left(\begin{array}{l}
A_{i 1}^{k} \\
E_{n 1}^{k}
\end{array}\right)=\left[\begin{array}{l}
h_{\tau 1}^{k} \\
h_{\nu 1}^{k}
\end{array}\right]
$$

Note that the matrix on the left hand side of (61) does not depend on the boundary value problem but only on the geometrical parameters. This can be an advantage in terms of numerical computation time decreasing. Finally, the remaining Fourier coefficients $F_{j}^{k}$ and $D_{n}^{k}$ can be computed respectively from equation (41) and (44).

\section{EXCITATION FORCES AND HYDRODYNAMIC COEFFICIENTS}

Once the scattering and/or radiation problem are solved which means that we determined the unknown Fourier coefficients for the orthogonal series, we know the velocity potential in the whole fluid domain. Then waves exciting forces and/or hydrodynamic coefficients can be determined by integrating the hydrodynamic pressure, given by the Bernoulli equation, over the wet surface of the body under consideration. From the linear wave theory the pressure is given by

$$
p(r, \theta, z, t)=-\rho \frac{\partial \Phi}{\partial t}=\mathrm{i} \omega \rho \phi(r, \theta, z) e^{-\mathrm{i} \omega t}
$$




\section{A. Added Mass and Radiation Damping Coefficients}

When the structure is moving, a radiation force acts on it and can be expressed as

$$
F_{q, j}^{k, i}(\omega)=\mathrm{i} \omega \rho \iint_{S_{i}} \phi_{q}^{k}(r, \theta, z) n_{j} \mathrm{~d} S
$$

where $F_{q, j}^{k, i}(\omega)$ is the complex representation of the radiation force acting on the body $i$ in the direction $j$ due to the motion of the body $k$ in the direction $q$ (here $q=3$ ). It is conventional to decompose this radiation force into two components, one proportional to the acceleration of body and the other proportional to his velocity as follows

$$
F_{q, j}^{k, i}(\omega)=-\omega^{2} \zeta_{q}\left[\mu_{q, j}^{k, i}+\frac{\mathrm{i}}{\omega} \lambda_{q, j}^{k, i}\right]
$$

where hydrodynamic coefficients $\mu_{q, j}^{k, i}$ and $\lambda_{q, j}^{k, i}$ are referenced in the literature as the added mass and the radiation or potential damping, respectively.

\section{B. Waves Excitation Forces in $z$ Direction}

When the structure is considered as fixed in the presence of an incident wave an excitation force acts on the bodies. Using a complex representation, excitation forces in the vertical direction can be expressed in terms of scattering potential, see (25), as

$$
f_{z}^{k}(\omega)=\mathrm{i} \omega \rho \iint_{S_{k}} \phi_{s}(r, \theta, z) n_{3} \mathrm{~d} S
$$

where $k$ indicates which body is under consideration and $n_{3}$ is the component of the generalised normal vector in $z$ direction.

\section{Numerical Results AND Discussion}

A specific code based on the above-presented formulation has been developped in order to solve problem (61). To carry out numerical computations, the infinite series in the expressions of the radiated and scattering potentials have to be truncated to a finite number of terms. According to the literature [2], [3], [5], it seems that considering only the first 30 terms shows good truncation characteristics. We have therefore chosen $N=30$ for potential expressions in all subdomains.

In order to validate the analytical expressions obtained for velocity potentials, several checks are done on numerical results and then different case-studies have to be considered. In Table I we have summarised geometrical configurations for each case-study where $\alpha$ is a variable parameter defined as $\alpha \in\left[\begin{array}{llllll}8 & 2 & 1 & .75 & .65 & .51\end{array}\right]$. Geometrical parameters for case study no. 1 and no. 3 are taken respectively from models developped by $\mathrm{Wu}$ et al. [7] and Chau and Yeung [8]. For all numerical computations we have used $h=1$ and for the scattering problem, solutions are obtained baised on the assumption of a unit amplitude incident wave, $A=1$.

First of all, we look at the matching of the velocity potential and its first derivative in the radial direction along the imaginary interfaces between subdomains. In Fig. 2 results are presented for the structure depicted in Section I for the
TABLE I

GeOMETRICAl PARAMETERS FOR NUMERICAL COMPUTATION

\begin{tabular}{l|cccccc}
\hline Case no. & $b / h$ & $R_{b} / h$ & $e_{1} / h$ & $e_{2} / h$ & $R_{p} / R_{b}$ & $R_{c} / R_{b}$ \\
\hline \hline 1 & 0.1 & 0.2 & 0.25 & 0.35 & $\alpha$ & $10^{-3}$ \\
2 & 0.1 & 0.2 & 0.25 & 0.35 & $\alpha$ & 0.5 \\
3 & 0.25 & 1 & 0.4 & 0.5 & $\alpha$ & 0.5 \\
\hline
\end{tabular}

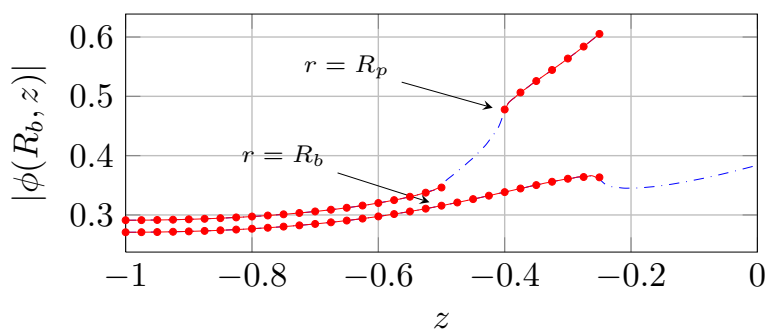

(a)

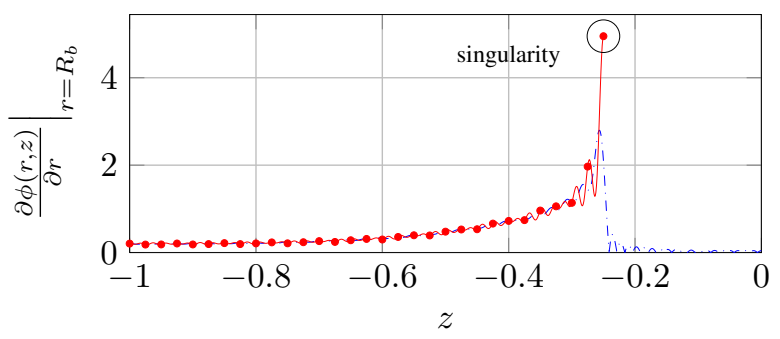

(b)

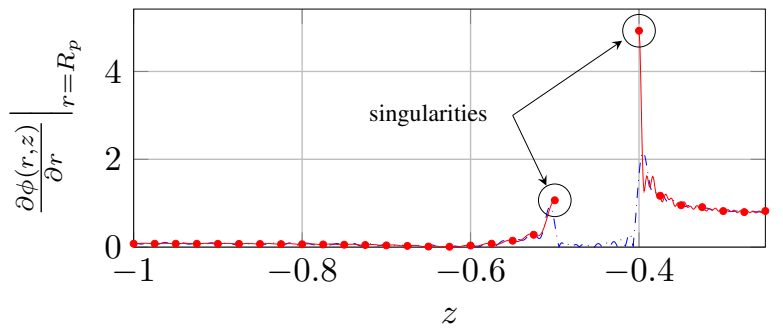

(c)

_... Ext. domain —. Int. domain

Fig. 2. Matching of the velocity potential $(a)$ and its first derivative $(b)$ and (c) for case no.3, $\alpha=.75$, at the imaginary interface $r=R_{b}$ and $r=R_{p}$ for $k_{e}=1$

case study no. 3 where the plate radius is smaller than that of the buoy, $\alpha=.75$, and where the buoy is moving and the platform is fixed. Excellent matching is achieved on velocity potential using numerical truncation given above. However for gradient visualisation purposes, we have used more terms in expressions to reduce the oscillations due to Bessel functions ( $N=80$ for regions $\Omega_{1}, \Omega_{2}$, and $N=40$ for regions $\Omega_{3}, \Omega_{4}$ ). Note the well-known singularity in Fig. $2(b)$ at the corner due to the discontinuous boundary condition (see [11] for more details).

Also one could use asymptotic behaviours of the presented model to compare results with existing models found in the literature, which indirectly verify the proposed expressions for potentials. Figures 3 to 5 show numerical results for 


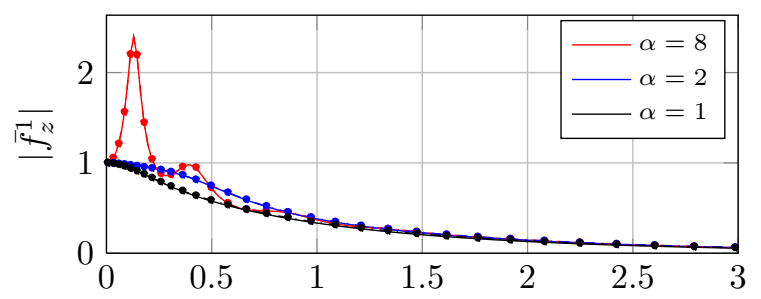

(a)

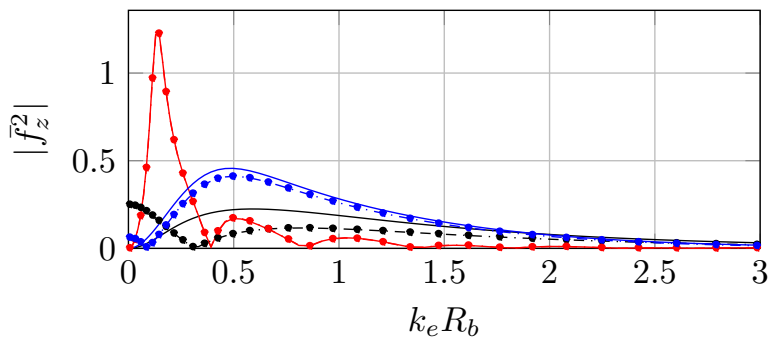

(b)

_ case no. 1 - c case no.2

Fig. 3. Dimensionless vertical forces, $(a)$ on buoy $(b)$ on platform.

hydrodynamic parameters when column radius tends toward zero, case-study no.1 for $\alpha=\left[\begin{array}{lll}8 & 2 & 1\end{array}\right]$. This configuration is similar to the model presented by Wu et al. in [7] (not shown). Same coefficients than those given in [7] have been used to non-dimensionnalise excitation forces in Fig. 3 and hydrodynamics coefficients in Figs. 4 and 5, and excellent correlation is found. Also parameters are shown for the case where the radius of the column is half of the radius of the buoy that corresponds to case-study no.2. Looking at added mass and radiation damping, we note that only the amplitude of the coefficients changes with the increase and decrease of the wet body surface but not the behaviour. Now, if we look at the excitation forces on the buoy, the presence of the column does not seem to have an effect on it. However, we notice that for the platform, the behaviour is totaly different at low frequency and we can observe a rebound which varies with the radius of the plate except for relatively big sizes. Then, it seems that a radius ratio between the plate and column exists for which the column no longer influences the behaviour of the wave excitation force on the platform. Figures 6 to 7 show numerical results for hydrodynamic parameters when plate radius tends toward column radius (case-study no. 3 for $\alpha=.51$ ) for two differents water depths $h=1$ and $h=5$. This configuration is similar to the model presented by Chau and Yeung in [8]. The same coefficients as above have been used to non-dimensionnalised excitation forces. For hydrodynamic coefficients, we divided by factor $\rho \pi R_{b}^{2}$ for the buoy and by factor $\rho \pi R_{c}^{2}$ for the platform. Here again figures show very good correlation between our model and the literature. Looking at the excitation forces on the platform Fig. 6 (b), one can notice that we do not have the behaviour described previously. Then it seems that a radius ratio between

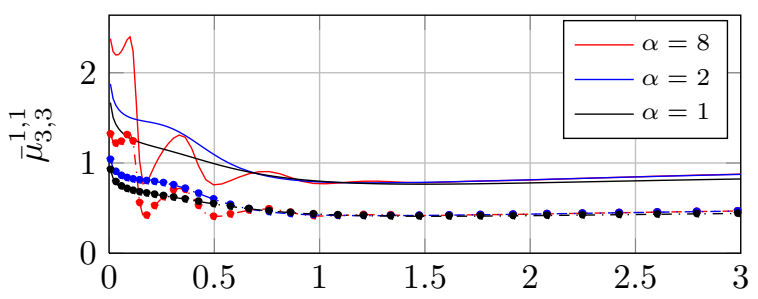

(a)

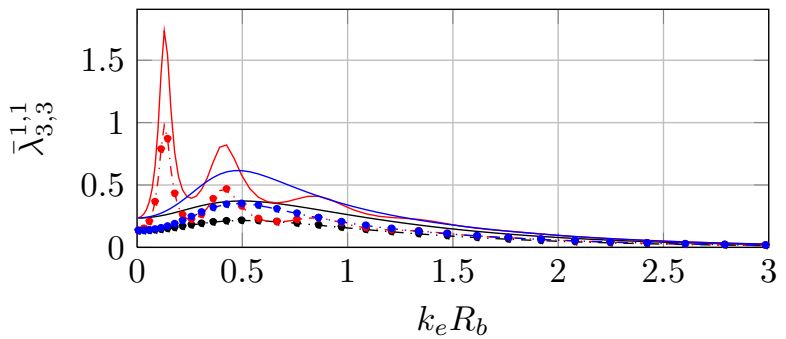

(b)

Fig. 4. Dimensionless added mass $(a)$ and radiation damping $(b)$ for the buoy.

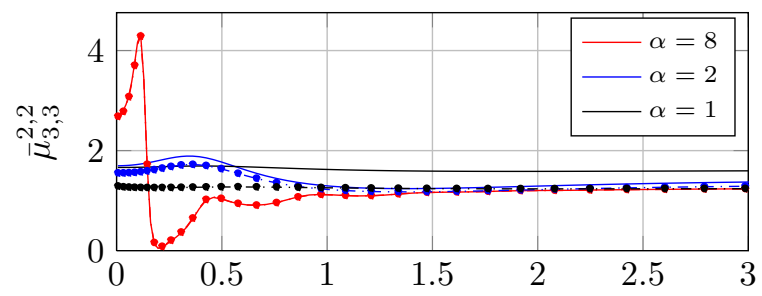

(a)

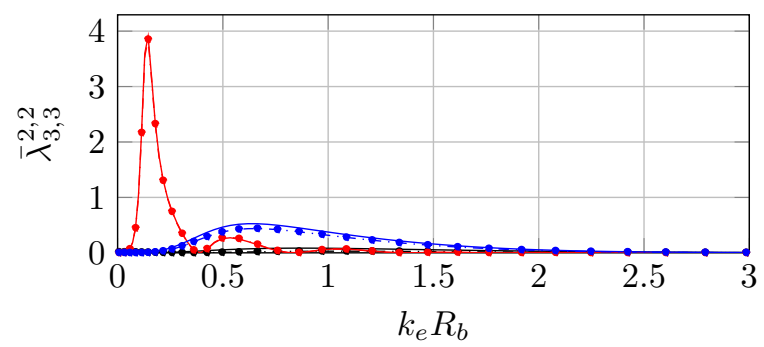

(b)

Fig. 5. Dimensionless added mass $(a)$ and radiation damping $(b)$ for the platform.

the plate and column exists for which the plate radius is not big enough to observe the rebound.

\section{CONCLUSION}

Based on the potential theory, a semi-analytical method has been presented in order to solve the radiation and scattering problem which provides hydrodynamic parameters in heaving mode for a specific wave energy converter. These parameters are particularly needed for designers to analyse the WEC 


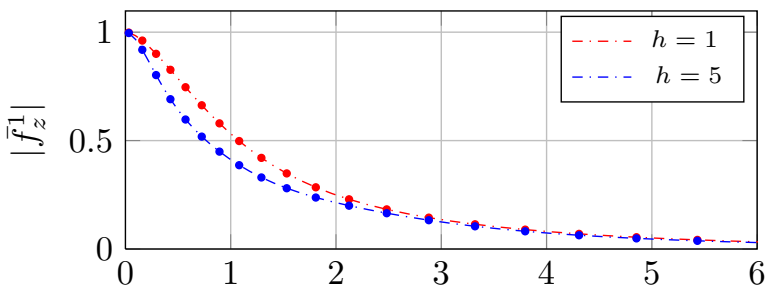

(a)

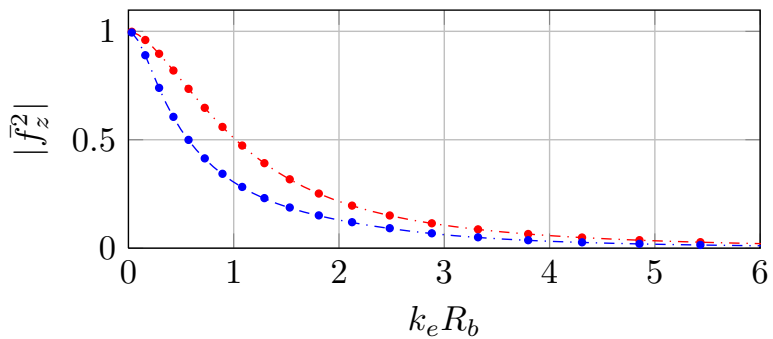

(b)

-..- $\alpha=.51$. Chau and Yeung [8]

Fig. 6. Dimensionless vertical forces on the buoy $(a)$, on the platform $(b)$.

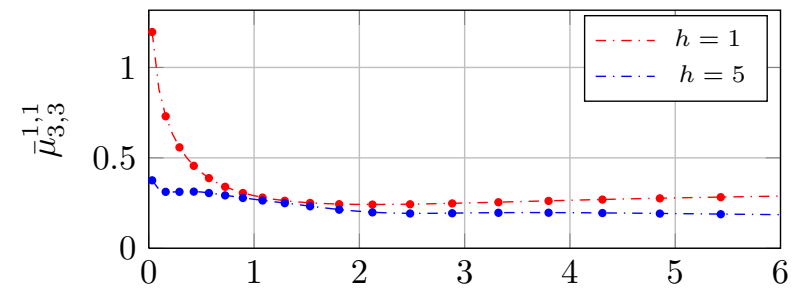

(a)

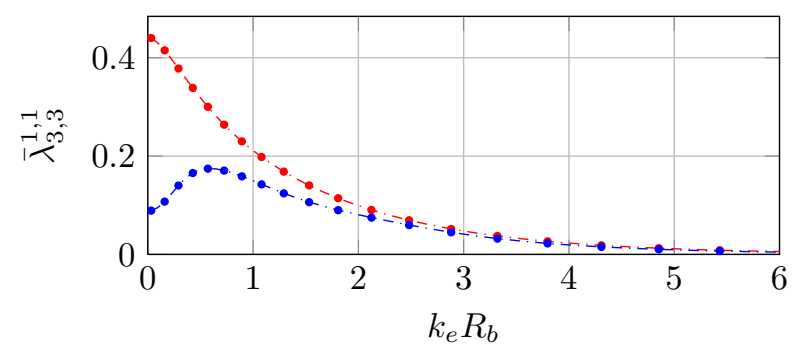

(b)

$\cdots \quad \alpha=.51 \cdot$ Chau and Yeung [8]

Fig. 7. Dimensionless added mass $(a)$ and radiation damping $(b)$ for the buoy.

dynamics in irregular waves. A specific code has been developped based on the mathematical formulations presented in this paper. For validation purposes, several numerical simulations have been carried out for different buoy, column, and plate radiuses. The results obtained have been compared to well-known and available models in the literature. The clearly confirm the appropriateness of the proposed semi-analytical approach.

\section{APPENDIX A}

$$
\text { EXPRESSION FOR } T_{n}(r), \tilde{T}_{n}(r)
$$

for $n=0$

$$
T_{0}=\frac{\ln \left(r / R_{c}\right)}{\ln \left(R_{x} / R_{c}\right)} \quad \tilde{T}_{0}=\frac{\ln \left(R_{x} / r\right)}{\ln \left(R_{x} / R_{c}\right)}
$$

for $n \geq 1$

$$
\begin{aligned}
& T_{n}=\frac{I_{0}\left(\gamma_{l} r\right) K_{0}\left(\gamma_{l} R_{c}\right)-I_{0}\left(\gamma_{l} R_{c}\right) K_{0}\left(\gamma_{l} r\right)}{I_{0}\left(\gamma_{l} R_{x}\right) K_{0}\left(\gamma_{l} R_{c}\right)-I_{0}\left(\gamma_{l} R_{c}\right) K_{0}\left(\gamma_{l} R_{x}\right)} \\
& \tilde{T}_{n}=\frac{I_{0}\left(\gamma_{l} R_{x}\right) K_{0}\left(\gamma_{l} r\right)-I_{0}\left(\gamma_{l} r\right) K_{0}\left(\gamma_{l} R_{x}\right)}{I_{0}\left(\gamma_{l} R_{x}\right) K_{0}\left(\gamma_{l} R_{c}\right)-I_{0}\left(\gamma_{l} R_{c}\right) K_{0}\left(\gamma_{l} R_{x}\right)}
\end{aligned}
$$

where $R_{x}=\min \left(R_{b}, R_{p}\right)$.

\section{ACKNOWLEDGMENT}

This work was supported in part by the Fonds Unique Interministériel (France) - Project "EM Bilboquet", in part by Région Bretagne, in part by Conseil Général du Finistère.

\section{REFERENCES}

[1] B. Molin, Hydrodynamique des structures offshore. Technip, 2002.

[2] L. Berggren and M. Johansson, "Hydrodynamic coefficients of a wave energy device consisting of a buoy and a submerged plate," Applied Ocean Research, vol. 14, no. 1, pp. 51-58, Jan. 1992.

[3] R. W. Yeung, "Added mass and damping of a vertical cylinder in finite-depth waters," Applied Ocean Research, vol. 3, no. 3, pp. 119-133, Jul. 1981.

[4] H. Eidsmoen, "Hydrodynamic parameters for a two-body axisymmetric system," Applied Ocean Research, vol. 17, no. 2, pp. 103-115, Jan. 1995.

[5] B.-J. Wu, Y.-H. Zheng, Y.-G. You, X.-Y. Sun, and Y. Chen, "On diffraction and radiation problem for a cylinder over a caisson in water of finite depth," International Journal of Engineering Science, vol. 42, no. 11-12, pp. 1193-1213, Jul. 2004

[6] B.-J. Wu, Y.-H. Zheng, and Y.-G. You, "Response Amplitude and Hydrodynamic Force for a Buoy over a Convex," Journal of Waterway, Port, Coastal, and Ocean Engineering, vol. 132, no. 2, pp. 97-105, Mar. 2006.

[7] B.-J. Wu, Y.-H. Zheng, Y. You, D. Jie, and Y. Chen, "On diffraction and radiation problem for two cylinders in water of finite depth," Ocean Engineering, vol. 33, no. 5-6, pp. 679-704, Apr. 2006.

[8] F. P. Chau and R. W. Yeung, "Inertia and Damping of Heaving Compound Cylinders," in Abstract for The 25th International Workshop on Water Waves and Floating Bodies, Harbin, China, 2010, pp. 1-4.

[9] C. Cochet and R. W. Yeung, "Two-Component AxisymmetricWaveEnergy Absorber - Analysis of Dynamics and Geometric Proportions," in The 27th International Workshop on Water Waves and Floating Bodies, Copenhagen, Denmark, 2012.

[10] S. A. Mavrakos, "Hydrodynamic coefficients in heave of two concentric surface-piercing truncated circular cylinders," Applied Ocean Research, vol. 26, no. 3-4, pp. 84-97, May 2004.

[11] C. Linton and P. McIver, Handbook of Mathematical Techniques for Wave/Structure Interactions, 1 st ed. Chapman and Hall/CRC, 2001. [Online]. Available: http://www.citeulike.org/group/8352/article/11022033

[12] J. Falnes, Ocean Waves and Oscillating Systems - Linear Interactions Including Wave-Energy Extraction. Cambridge University Press, Apr. 2002.

[13] D. D. Bhatta and M. Rahman, "On scattering and radiation problem for a cylinder in water of finite depth," International Journal of Engineering Science, vol. 41, no. 9, pp. 931-967, May 2003. 